\title{
Simulation Analysis for Opening Performance of Medium Voltage Vacuum Circuit Breaker Based on ADAMS and Maxwell
}

\author{
Ding Xianliang, Wang Zhiming , Yin Qinlin, Chen Pengwei, Zhou Shijin and Yang Yang \\ School of Mechanical Engineering, Nanjing University of Science and Technology, Nanjing 210094, China
}

\begin{abstract}
The circuit breakers play a important role in control and protect the power system and the vacuum circuit breaker has been widely used in the field of medium voltage with its excellent opening performance. Virtual prototyping technology is also became more and more popular in design and optimization of the vacuum circuit breaker.In this paper, the electromagnetic simulation software Ansoft Maxwell is used to analyze the electric repulsion of the circuit breaker in the case of open the rated short circuit breaking current. The 3D model that was built by CREO is imported into ADAMS .Then constraints, contact force, and the electric repulsion force that was analysezed in Ansoft Maxwell is added into the 3D model. Therefore, we can carry on the multi-body dynamics simulation to the 3D model. Then We can get the opening performance of the vacuum circuit breaker in the condition of open circuit rated short circuit breaking current. The simulation results show that the circuit breaker can still meet the performance requirements in the condition of open circuit rated short circuit breaking current.
\end{abstract}

\section{Introduction}

With the rapid development and popularization of virtual prototyping technology, more and more engineering designers begin to design and develop the products using computer. The virtual prototyping technology is more and more popular all over the world. The vacuum circuit breakers have been widely used in the medium voltage field, because it has excellent performance. The engineering and technical personnel have already begun to apply the virtual prototyping technology to design and develop the vacuum circuit breaker. For example, Tu Kai [1] and others use Pro/E to build the 3D model and then import it to the ADAMS. Finally they simulate and analyze the mechanical system of the circuit breaker, which provides a good platform for the design and optimization of circuit breaker. Zhou Yinzheng [2] and others use ADAMS software to analysis the closing bounce of vacuum circuit breaker, which to study the influence of the preload of the contact spring to the closing time. But both they only analyzed the mechanical performance of the circuit breaker under the no-load state, and not consider that there is a big electric repulsion force between the static contact and the moving contact in the condition of open the rated short-circuited breaking current, which affect the opening performance of the circuit breaker. In this paper, the model of the arc chamber is built by Ansoft Maxwell, and analyze the electric repulsion in the process of opening in the case of open the rated short circuit breaking current .The 3D model of ZN63A-12 vacuum circuit breaker was established by Creo and then is imported into ADAMS, at the same time import the electric repulsion which was analyzed in Ansoft Maxwell into ADAMS. After add constraints and contact force, we can carry on multi body dynamics simulation analysis. The finally we can get the opening performance of the vacuum circuit breaker in the case of the rated short-circuited breaking current.

\section{Electric repulsion analysis and simulation}

Vacuum arc extinguishing chamber is the core component of vacuum circuit breaker. And its main function is to cut off the power quickly and extinguish the arc after cutting off the power of high voltage circuit suddenly, which can avoid the occurrence of accidents. This function comes true better because of the good insulation of the vacuum. During the opening, an arc will generate between the contacts and transfer current between contacts, before the contacts contact with each other. Current changes will produce a magnetic field, which will produce electric repulsion force in the process of opening. The size of the electric repulsion relate on not only circuit configuration and contact materials loop structure, but also the size of the current. Ansoft Maxwell is a 2D/3D electromagnetic field finite element analysis software. Power、voltage and magnetic can be solved in the electrostatic field、static magnetic field time-varying electric field magnetic field、 eddy current field transient field and temperature field .It can be used to analyze the characteristic of static 、 steady state and transient state of the transformer、motor 
and permanent magnet device which work in normal condition and abnormal condition and so on.

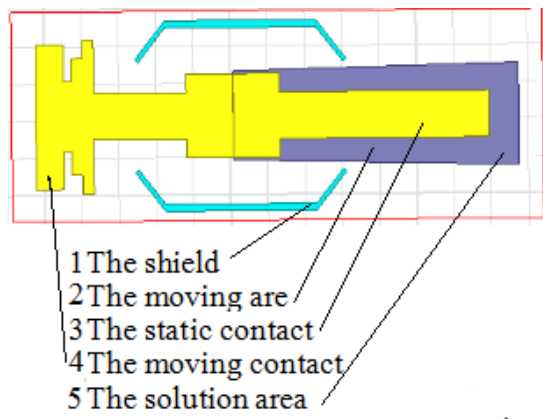

Figure 1. The 2D model of interrupter.
In this paper, the ZN63A-12 type vacuum circuit breaker is studied. As is shown in Figure 1, the 2D structure of the arc chamber is built in Ansoft Maxwell [3], which is mainly composed of the static contact、 the moving contact v the shield and the solution area. Then we should define the material of each parts. The moving contact、 the static contact and the shield are covered for the copper material, and others are vacuum. Then we should define the current between contacts. Because the rated short-circuited current is very complicated in the course of opening, so we import the rated short-circuited breaking current that we measured. As shown in Figure 2, it's the figure that the rated short-circuited breaking current changes with time.

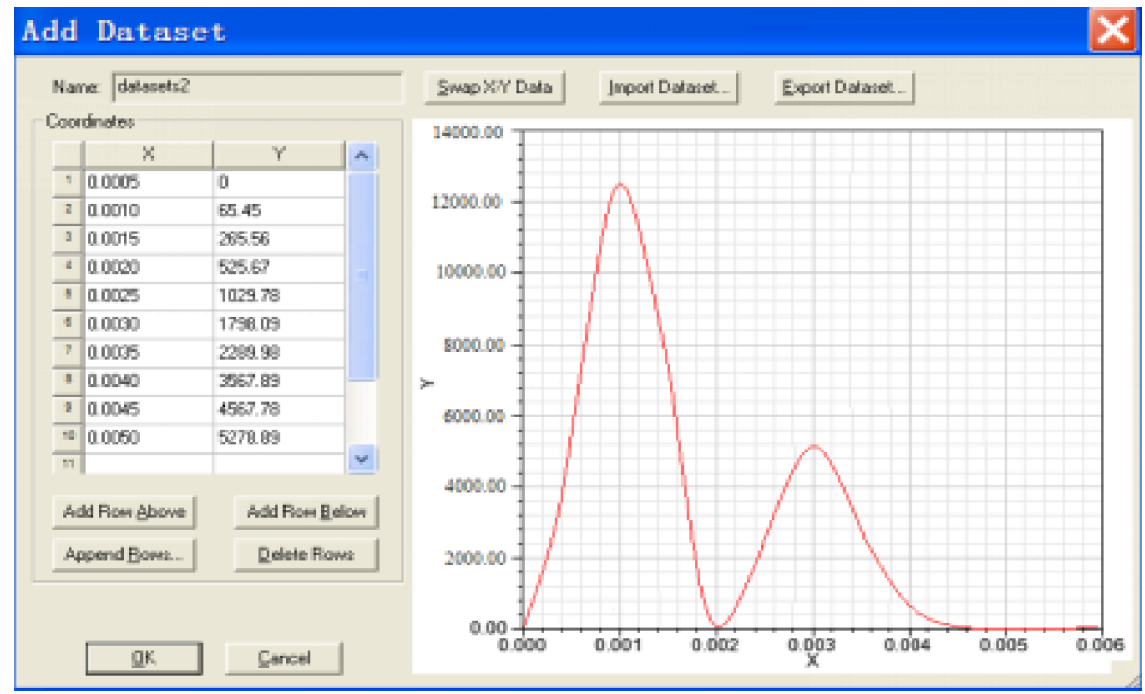

Figure 2. The rated short-circuited breaking current.

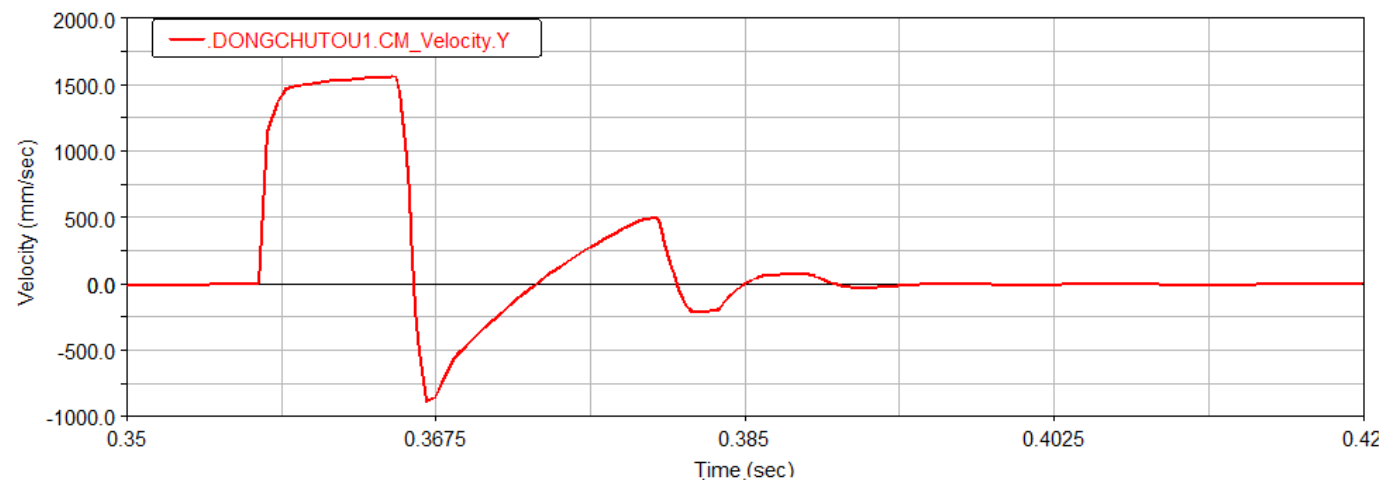

Figure 3. The velocity curve of the moving contact.

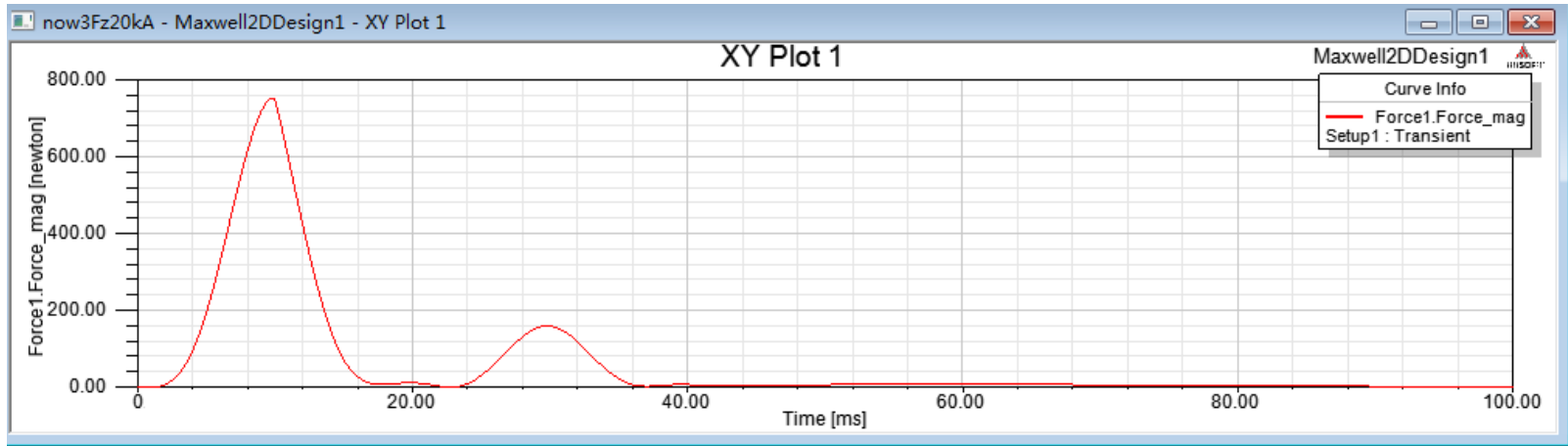

Figure 4. The picture of electric repulsion. 
Next we should import the velocity of the moving contact. In order to ensure the reliability of the simulation results, we import the velocity curve of the moving contact in the ADAMS simulation under the no-load condition. As shown in Figure 3, it's the velocity curve of the moving contact in this case.

Finally after all the steps have been done, we can start the simulation analysis. And after simulate analysis, electric repulsion can be obtained like Figure 4. The maximum value of the electric repulsion is about $780 \mathrm{~N}$ when the rated short-circuited breaking current reaches the maximum value.

\section{Dynamic model establishment and simulation}

The vacuum circuit breaker [4] is mainly composed of the spring operating mechanism, the vacuum arc extinguishing chamber, the transmission mechanism, the frame and so on. In order to analyze the opening performance of the vacuum circuit breaker, we need to establish the 3D model and import it into the ADAMS. Then we can carries on the dynamics simulation analysis to the $3 \mathrm{D}$ model.

\subsection{Establish the 3D model}

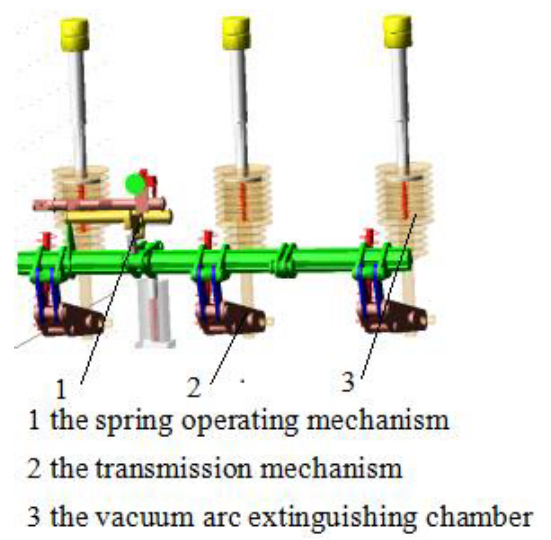

Figure 5. The 3D model of the vacuum circuit breaker.

Each component of ZN63A-12 type vacuum circuit breaker is established by $3 \mathrm{D}$ modeling software CREO. In order to simplifying the model, we should reduce some holes , bolts and racks, which have no impact on the simulation analysis. It can reduce the constraints that we need add into the dynamic simulation and shorten the time to draw and simulate. Finally, the final the model is finished in the assembly module and is imported into ADAMS.As shown in Figure 5, the 3 model of the vacuum circuit breaker is mainly composed of the vacuum arc extinguishing chamber, the transmission mechanism, and the spring operating mechanism. When the power system is in trouble, the circuit breaker will receive the signal and the spring operating mechanism will respond quickly and drive the moving contact to carry on the opening operation. At last, the vacuum circuit breaker achieve opening operation, cut off the current and prevent accidents quickly. So as to ensure the safe operation of the system, The main performance parameters of the circuit breaker are as follows. Average opening velocity $1.1 \pm 0.2 \mathrm{~m} / \mathrm{s}$; Opening time $20 \sim 50 \mathrm{~ms}$; Contact wear thickness $\leq 3 \mathrm{~mm}$.

\subsection{Simulation setup and analysis}

ADAMS is the most outstanding mechanical system simulation software which is developed by the Mechanical Dynamic Inc. It is also the most authoritative and widely used mechanical system dynamics analysis software and has a the highest of occupancy in the global [5-6]. Firstly, we must add constraints to the various components of the $3 \mathrm{D}$ model. The static contact is added to the fixed constraint, the moving contact is added to the translation constraint and the appropriate constraints are added to others. Secondly, we should add the closing spring, the opening spring and the contact spring, which stiffness coefficients are $70 \mathrm{~N} / \mathrm{mm}, 20 \mathrm{~N} / \mathrm{mm}$, and $100 \mathrm{~N} / \mathrm{mm}$. And The stiffness coefficients and preload of all springs are shown in Table 1. Thirdly, the electric repulsion which was analyzed in the Ansoft Maxwell should be imported to ADAMS and added on the moving contact. Fourthly, we need add force to the parts which contact with each other, especially the force between the moving contact the static contact and the brake keep pawl. The stiffness coefficient is set to $1 \times 10^{5} \mathrm{~N} / \mathrm{mm}$; the Impact index is set to 1.5 ; the damping coefficient is set to $50 \mathrm{~N}-\mathrm{sec} / \mathrm{mm}$ and the cutting depth is set to $0.1 \mathrm{~mm}$. Finally, we need to apply a rotating drive on the split gate.

Table 1. The stiffness coefficients of all springs.

\begin{tabular}{ccc}
\hline name & $\begin{array}{c}\text { The stiffness } \\
\text { coefficients }\end{array}$ & Preload \\
\hline The contact spring & $100 \mathrm{~N} / \mathrm{mm}$ & $2200 \mathrm{~N}$ \\
The opening spring & $20 \mathrm{~N} / \mathrm{mm}$ & $-600 \mathrm{~N}$ \\
The buffer spring & $5.0 \mathrm{~N} / \mathrm{mm}$ & $30 \mathrm{~N}$ \\
The closing latch spring & $3 \mathrm{~N} / \mathrm{mm}$ & $300 \mathrm{~N}$ \\
The closing coil spring & $2 \mathrm{~N} / \mathrm{mm}$ & $200 \mathrm{~N}$
\end{tabular}

\subsection{The analysis of the simulation result}

We need select the appropriate simulation time and step size and begin to simulate the dynamic model. The simulation results will be processed after simulation, we can get the velocity time curve and the position time curve of the moving contact from figure 6 and figure 7. As is shown by the analysis and simulation, at the initial stage of the opening operations, the velocity of the moving contact is rapidly increased to the maximum value under the joint action of the contact spring and the opening spring, and then the velocity will slow down. When collision with the split opening buffer, the velocity will rapidly drop to zero, and then moving contact will be slowly elastic vibration until it is completely stationary. The whole process takes about $35.5 \mathrm{~ms}$ and the time limit of the opening operation is between $20 \mathrm{~ms}$ to $50 \mathrm{~ms}$, so it fully meets the time requirements. The average opening velocity is $110.3 \mathrm{~mm} / \mathrm{s}$ which is meet the velocity requirements of opening operations .So the vacuum circuit breaker can also meet the performance requirements when open the rated short-circuited current. 


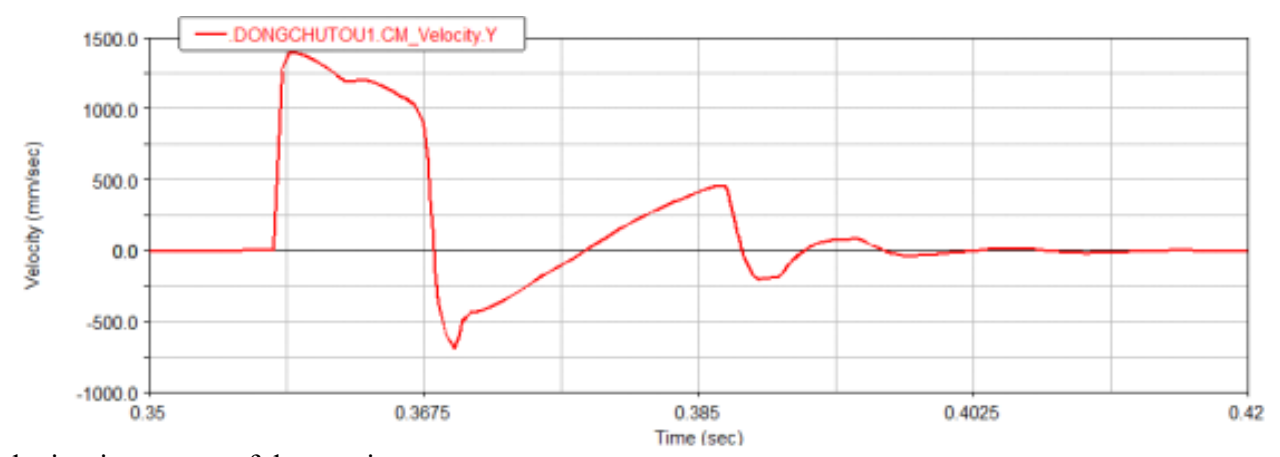

Figure 6. The velocity time curve of the moving contact.

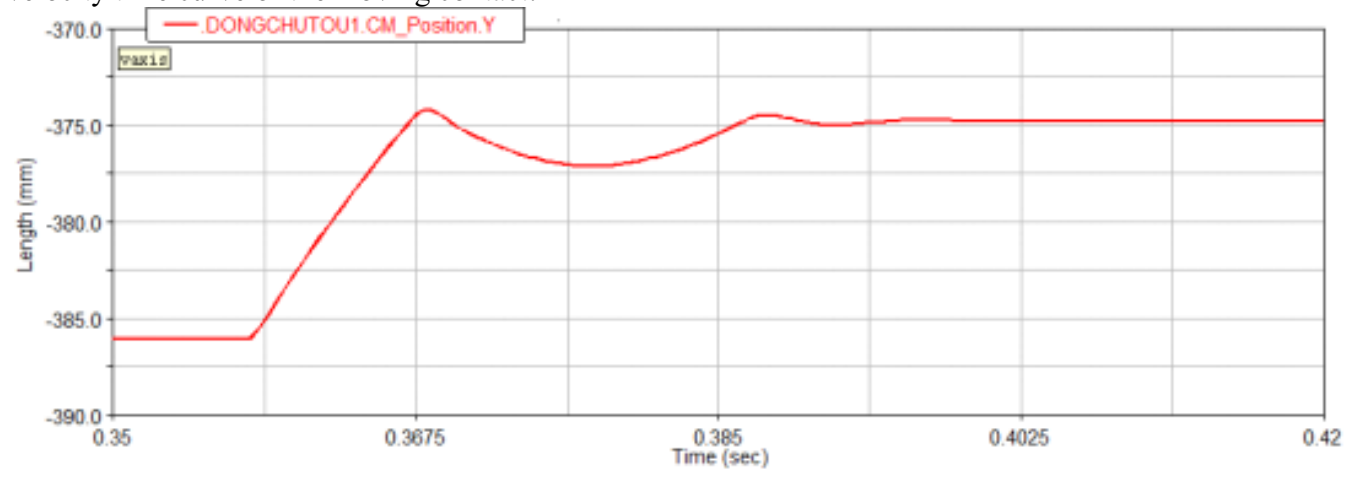

Figure 7. The position time curve of the moving contact.

\section{Conclusion}

With the development of the virtual prototyping technology, more and more engineering and technical personnel use the computer to carry out product design and optimization, which can not only greatly shorten the cycle of the product development and design, but also reduce the cost of development. Along with the development of CAE technology, the application of the simulation technology is more and more comprehensive and the simulation results are more and more reliable. In this paper, the $2 \mathrm{D}$ model of the vacuum arc extinguishing chamber is built in Ansoft Maxwell, and then we need define parameters of materials and set incentive of the model so that the electric repulsion is solved in the process of opening operation. The 3D model of ZN63A-12 vacuum circuit breaker is established by CREO and imported into ADAMS and then we need set the constraints, the contact force and the stiffness coefficients of all springs to the model and import the electric repulsion which was simulated in the Ansoft Maxwell to model. Finally we will simulate the opening process of the vacuum circuit breaker. The simulation results show that the circuit breaker can meet the requirements in the conditions of open the rated short-circuited current. The analysis results can provide reference for the further design and optimization of vacuum circuit breaker.

\section{References}

1. Tu Kai, Zhou Yi, Zhang Li. Simulation analysis of high voltage circuit breaker mechanism with ADAMS and Pro/E[A]. Machine research and Application,49(2009).

2. Zhou Yinzheng,Lu Min, Zhuang Huogeng. The analysis of the trip of vacuum circuit breaker based on ADAMS[A]. Low voltage electrical apparatus, 14(2014).

3. Zhao Bo, Zhang Hongliang. Application of Ansoft 12 in engineering electromagnetic field [M]. Beijing: China Water Conservancy and Hydropower Press, 38(2010).

4. Li Jianji, High voltage circuit breaker and its application ,30(2004).

5. Chen Fenghua. The virtual prototyping technology ADAMS 2012 from entry to the master [M]. Beijing: Tsinghua University press, 18(2013).

6. Chen Demin, Chuang Fenghuai, Zhang Ketao. Proficient in Virtual Prototyping Technology ADAMS 2005/2007 [M]. Beijing: Chemical Industry Press, 207(2010). 